\title{
The Role of Brown Adipose Tissue in the Development and Treatment of Nonalcoholic Steatohepatitis: An Exploratory Gene Expression Study in Mice
}

Authors

Toon J.I. De Munck1, 2 *, Pan Xu1, 2 *, Brechtje L.J. Vanderfeesten ${ }^{1}$, Montserrat Elizalde², Ad A.M. Masclee ${ }^{1,2}$, Frederik Nevens ${ }^{3}$, David Cassiman ${ }^{3}$, Frank G. Schaap², 4, 5, Daisy M.A.E. Jonkers ${ }^{1,2}$, Jef Verbeek ${ }^{3}$

Affiliations

1 Division of Gastroenterology and Hepatology, Department of Internal Medicine, Maastricht University Medical Centre, Maastricht, The Netherlands

2 School of Nutrition and Translational Research in Metabolism (NUTRIM), Maastricht University, The Netherlands

3 Department of Gastroenterology and Hepatology, University Hospitals KU Leuven, Leuven, Belgium

4 Department of Surgery, Maastricht University Medical Centre, Maastricht, The Netherlands

5 Department of General, Visceral and Transplantation Surgery, RWTH University Hospital Aachen, Aachen, Germany

Key words nonalcoholic fatty liver disease NAFLD Nrg4 Ucp1 obesity

received $\quad 14.04 .2020$

accepted $\quad 21.10 .2020$

Bibliography

Horm Metab Res 2020; 52: 1-8

DOI 10.1055/a-1301-2378

ISSN 0018-5043

(c) 2020. Thieme. All rights reserved.

Georg Thieme Verlag KG, Rüdigerstraße 14,

70469 Stuttgart, Germany

Correspondence

Jef Verbeek MD, PhD

Department of Gastroenterology and Hepatology University Hospitals KU Leuven

Herestraat 49

Leuven

Belgium

Tel.: + 32163442 25, Fax: $\mathbf{\square}$

jef.verbeek@uzleuven.be
\# Supplementary Material is available under https://doi. org/10.1055/a.1301-2378.

\section{ABSTRACT}

Brown adipose tissue (BAT) might be a beneficial mediator in the development and treatment of nonalcoholic steatohepatitis (NASH). We aim to evaluate the gene expression of BAT activity-related genes during the development and the dietary and surgical treatment of NASH. BAT was collected from male C57BL/6] mice that received a high fat-high sucrose diet (HFHSD) or a normal chow diet (NCD) for 4 and 20 weeks ( $n=8-9$ per dietary group and timepoint) and from mice that underwent dietary intervention (return to NCD) $(n=8)$, roux-en-y gastric bypass (RYGB) $(n=6)$, or sham procedure $(n=6)$ after 12 weeks HF-HSD. Expression of BAT genes involved in lipid metabolism ( $C d 36$ and $C p t 1 b ; p<0.05)$ and energy expenditure (Ucp1 and Ucp3; $p<0.05$ ) were significantly increased after 4 weeks HF-HSD compared with NCD, whereas in the occurrence of NASH after 20 weeks HF-HSD no difference was observed. We observed no differences in gene expression regarding lipid metabolism or energy expenditure at 8 weeks after dietary intervention (no NASH) compared with HF-HSD mice (NASH), nor in mice that underwent RYGB compared with SHAM. However, dietary intervention and RYGB both decreased the BAT gene expression of inflammatory cytokines (Il1b, Tnf- $\alpha$ and MCP-1; $\mathrm{P}$ 0.05). Gene expression of the batokine neuregulin 4 was significantly decreased after 20 weeks HF-HSD $(p<0.05)$ compared with NCD, but was restored by dietary intervention and RYGB ( $p<0.05)$. In conclusion, BAT is hallmarked by dynamic alterations in the gene expression profile during the development of NASH and can be modulated by dietary intervention and bariatric surgery.

\footnotetext{
* Co-first authors
} 


\section{Introduction}

Nonalcoholic fatty liver disease (NAFLD) is the most common cause of liver disease in the Western world, with a prevalence of $20-30 \%$ in the general adult population [1]. NAFLD is strongly associated with the metabolic syndrome and may promote the development of type 2 diabetes and cardiovascular diseases [2]. Its progressive subtype nonalcoholic steatohepatitis (NASH) can progress to liver fibrosis and ultimately to cirrhosis, liver failure, and hepatocellular carcinoma [3]. Now already, NASH is the second most common indication for liver transplantation in the United States, and it has been projected to become the major cause of liver related morbidity and mortality as well as the leading indication for liver transplantation within the next decade [4].

Up to now, no pharmacological therapy has been approved for NASH despite significant scientific efforts [5]. This can partly be explained by the complex pathophysiology of NASH, which is the result of an interplay of various mechanisms in different metabolic tissues and organs throughout the body such as the gut, muscle and adipose tissue [6]. In this perspective, the potential beneficial role of brown adipose tissue (BAT) in NASH has gained interest in the last years [7]. BAT uses exogenous glucose and fatty acids to generate heat in their abundant mitochondria that are characterized by the unique expression of uncoupling protein 1 (Ucp1) [8]. In this way, BAT increases energy expenditure through heat production. In addition to these metabolic fluxes, BAT can secrete regulatory molecules, also called "batokines", including neuregulin 4 (Nrg4) and fibroblast growth factor 21 (Fgf21) that might directly impact pathophysiological processes in the liver related to NASH $[9,10]$.

The recent discovery of BAT in human adults has raised the hope that BAT can be therapeutically targeted in obesity-associated metabolic diseases including NASH [11]. Several studies showed a significant negative correlation between BAT activity and body mass index $[12,13]$. One study using PET-CT showed an inverse correlation between BAT activity and NAFLD prevalence in adult individuals.[14] In a foz/foz NASH mice model beta-3 adrenergic receptor (b3-AR) agonists restored BAT function, improved glucose tolerance, and reduced hepatic lipid content but had no impact on hepatic inflammation [11]. In humans, the b3-AR agonist mirabegron induced a dose dependent increase BAT activity [15].

However, little is known about the changes in BAT function throughout the development of NASH nor the contribution of BAT to beneficial metabolic effects of dietary intervention and bariatric surgery, the two only effective treatment strategies for NASH at the moment. Studies to assess molecular changes in BAT in humans, in particular with a longitudinal set-up, are not feasible because of the need of surgical intervention to obtain BAT.

Therefore, in this exploratory study, we performed a gene expression analysis of BAT in a 'Western' high fat-high sucrose diet (HF-HSD)-induced mouse model of NASH that closely resembles the hepatic and systemic metabolic profile of typical NASH patients [16-18]. This model allowed us to study the longitudinal molecular changes in BAT during the development of NASH. In addition, we analyzed BAT gene expression changes after dietary intervention and roux-en-y gastric bypass (RYGB) in mice with pre-existent NASH.

\section{Materials and Methods}

\section{Animals, diet, and experimental set-up}

Ethical approval was obtained from the animal welfare committee of the University of Leuven (protocol number: P088/2011) [18]. In all experiments male C57BL/6] mice (The Jackson Laboratory, Bar Harbor, Maine, USA) were housed under a 14-hour light/10-hour dark cycle at $21-23{ }^{\circ} \mathrm{C}$ with ad libitum access to water. A high fathigh sucrose diet (HF-HSD) was used with $44.6 \%$ of kcal derived from fat (of which $61 \%$ saturated fatty acids) and $40.6 \%$ of kcal derived from carbohydrates (primarily sucrose $340 \mathrm{~g} / \mathrm{kg}$ diet) (TD.08811, 45\% kcal Fat Diet, Harlan Laboratories Inc., Madison, Wisconsin, USA) or a normal chow diet (NCD) as control (V1534000 ssniff R/M-H, ssniff Spezialdiäten $\mathrm{GmbH}$, Soest, Germany). Each individual mouse used in the current study has already been extensively phenotyped with respect to liver histology (histological scores of steatosis, hepatocyte ballooning, lobular inflammation and fibrosis are presented in $>$ Table 1S), hepatic molecular changes, fat distribution, glucose homeostasis and plasma parameters. Our group published these data in two separate manuscripts $[17,18]$. In experiment $A$ ( $n=8$ per group), mice received a HF-HSD or NCD as control for 4 weeks ( $\triangleright$ Fig. 1). Four weeks HF-HSD led to a significant increase in bodyweight, total fat mass and glucose intolerance compared to NCD mice, but did not lead to significant histological alterations or NASH ( $>$ Table 1S) [17]. In experiment $B$, BAT was collected from mice that received a HF-HSD for 20 weeks $(n=9)$ or NCD $(n=9)$ as control and from mice that underwent a dietary intervention (i. e. switch from HF-HSD to NCD) $(n=8)$ after 12 weeks HF-HSD (> Fig. 1) [18]. Mice that received 20 weeks HFHSD displayed obesity, glucose intolerance, insulin resistance and NASH in contrast to NCD mice that had normal liver histology $[16,18]$. As previously reported, twelve weeks of HF-HSD already induced the complete spectrum of NASH with steatosis, ballooning, hepatocyte inflammation and fibrosis, which together with the obesity and glucose intolerance completely normalized after the applied dietary intervention $[17,18]$. In experiment $C$, roux-en-y gastric bypass (RYGB) $(n=6)$ or SHAM $(n=6)$ operation was performed in mice that in advance were fed HF-HSD for 12 weeks to induce the phenotype of $\mathrm{NASH}$, obesity and insulin resistance ( $\triangleright$ Fig. 1) [17]. After the procedure both groups received an HFHSD for an additional 8 weeks. RYGB decreased the body weight, improved hepatic steatosis and inhibited the progression of inflammation, resulting in a significantly lower NAFLD activity score (NAS) in RYGB mice compared to SHAM mice [17].

\section{Sacrifice and plasma analysis}

After a 6-hour fasting period, mice were anesthetized with sodium pentobarbital (intraperitoneal injection, $50 \mathrm{mg} / \mathrm{kg}$ body weight) and killed by blood sampling via cardiac puncture. Plasma was obtained by centrifugation of blood (6000 rpm for 5 min at $4{ }^{\circ} \mathrm{C}$ ) that was collected in heparinized syringes [17]. Plasma Tnf- $\alpha$ levels were determined by ELISA according manufacturer's instructions (STEMCELL Technologies Inc., Vancouver, Canada). Interscapular BAT of all mice was collected by surgical excision. The dissected adipose tissue was weighed, snap frozen in liquid nitrogen and stored at $-80{ }^{\circ} \mathrm{C}$ until further molecular analyses. 


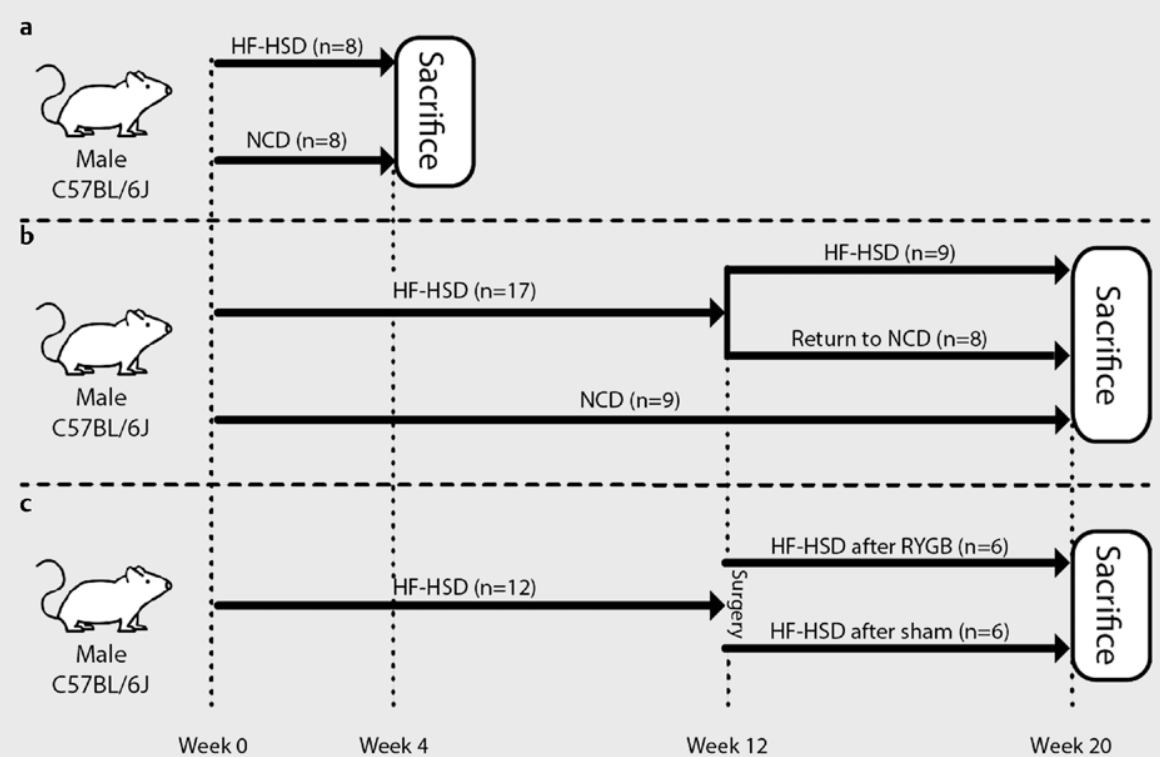

- Fig. 1 Experimental set-up of the mouse model. Experiment a: mice were fed a high fat-high sucrose diet (HF-HSD) for 4 weeks or a normal chow $\operatorname{diet}(N C D)$ as control. Experiment B: mice were fed a HF-HSD for 12 weeks $(n=18)$, and then randomized to a group that underwent continuation of HF-HSD for 8 weeks $(n=9)$ or a group of which the HF-HSD was switched to NCD for 8 weeks $(n=9)$, representing the dietary intervention. 9 mice received NCD during the entire experiment of 20 weeks. Experiment C: mice were fed a HF-HSD for 12 weeks $(n=12)$, and then randomized to a group that underwent a SHAM $(n=6)$ or RYGB $(n=6)$ operation. After the operation all mice were fed a HF-HSD diet for 8 weeks.

\section{RNA isolation and quantitative RT-PCR}

Tissue samples of BAT of the mice were homogenized, phase separation was performed, and total RNA was isolated using the Trizol (Ambion, life technologies, Carlsbad, USA) method according to the manufacturers' protocols. RNA was further purified with the RNeasy Mini Kit (Kit 74104, Qiagen, Hilden, Germany). RNA quantification and purity was evaluated with Nanodrop (Thermo Scientific, Waltham, USA). Five micrograms of RNA were reverse transcribed into cDNA using MultiScribe reverse transcriptase and random primers (Kit 4368814, Applied Biosystems, Vilnius, Lithuania). The PCR reaction was performed in a mixture that contained appropriate forward and reverse primers and the iQ SYBR Green Supermix (Bio-Rad Laboratories, Hercules, USA) as the indicator. Real-time PCR amplification and data analysis were performed using the CFX96 Touch Real-Time PCR Detection System (Bio-Rad Laboratories, Hercules, USA). Genes were assayed in triplicate for each sample in a High-Profile 96-Well PCR Plate (Bio-Rad Laboratories, Hercules, USA). The data were analyzed using the $\Delta$ Ct-method and normalized to internal housekeeping gene control of cyclophilin A mRNA to determine relative gene expression levels (mean expression level in the respective control group was set to 1.0). All primer sequences used are shown in $>$ Table $\mathbf{2 S}$.

\section{Statistical analysis}

Statistical analyses were performed using GraphPad Prism version 6 (GraphPad Software). Normality was tested with the D'Agostino-Pearson omnibus K2 normality test. Statistical differences between two groups were assessed by either unpaired $t$-test (parametric data) or Mann-Whitney test (nonparametric data). Statistical differences between three groups were assessed by one-way
ANOVA and Tukey post-hoc analysis (parametric data) or KruskalWallis and Dunn's multiple comparisons test (nonparametric data). A p-value of $<0.05$ was considered as statistically significant. Significance was represented by: ${ }^{*} p<0.05$, and ${ }^{*}{ }^{*} p<0.01$. Error bars are standard error of mean (SEM).

\section{Results}

\section{Four Weeks HF-HSD increases the expression of genes related to lipid metabolism and energy expenditure in BAT, while these changes are lost after 20 weeks HF-HSD}

After 4 weeks HF-HSD (no NASH), cluster of differentiation 36 (Cd36) mRNA, a gene involved in fatty acid uptake into BAT [19], and carnitine palmitoyltransferase $1 \mathrm{~b}$ (Cpt1b) mRNA, a gene involved in fatty acid trafficking into the mitochondria [20], were significantly increased compared with their levels in NCD mice (normal liver histology) ( $\$$ Fig. 2). This increase in gene expression was not present after 20 weeks HF-HSD (NASH) compared to NCD mice. Moreover, HF-HSD targets genes involved in mitochondrial function. An increased uncoupling protein 1 (Ucp1) and uncoupling protein (Ucp3) mRNA in BAT after 4 weeks HF-HFD (no NASH) were observed compared with NCD ( $\triangleright$ Fig. 2), but no significant differences were seen after 20 weeks HF-HSD feeding (NASH) compared with 20 weeks NCD feeding. lodothyronine deiodinase 2 (Dio2), a gene that promotes non-shivering thermogenesis in BAT by converting inactive thyroxine (T4) into active thyroid hormone (T3) [21] did not significantly differ between the dietary groups at any time point (HF-HSD compared to NCD at 4 and 20 weeks). Further- 


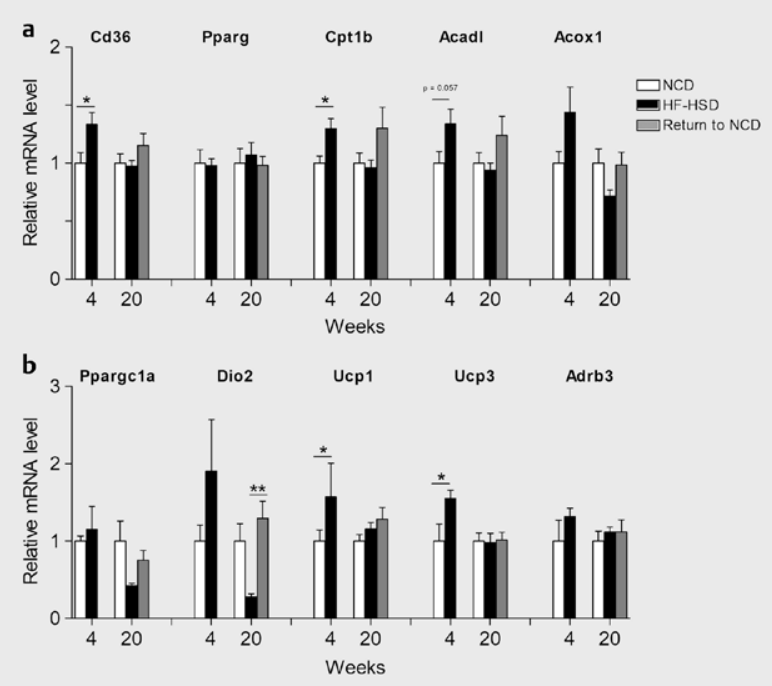

- Fig. 2 Effect of 4 and 20 weeks HF-HSD and dietary intervention on genes related to BAT activity. a mRNA levels of genes involved in fat metabolism: Cluster of Differentiation $36(\mathrm{Cd} 36)$, peroxisome proliferator-activated receptor gamma (Ppary), Carnitine palmitoyltransferase 1b (Cpt1b) and the lipid beta-oxidation genes acyl-Coenzyme A dehydrogenase, long-chain (Acadl) and acyl-Coenzyme A oxidase 1 (Acox1). b mRNA levels of genes involved in energy expenditure: peroxisome proliferative activated receptor, gamma, coactivator 1 alpha (Ppargc1 $\alpha$ ), lodothyronine Deiodinase 2 (Dio2), Uncoupling Protein 1 (Ucp1), Uncoupling Protein 3 (Ucp3), adrenergic receptor beta 3 (Adrb3). ${ }^{*} \mathrm{p}<0.05$ and ${ }^{* *} \mathrm{p}<0.01$. Data are presented as mean. Error bars are standard error of mean (SEM). 4 weeks: $\mathrm{n}=8$ for chow and HF-HSD groups. 20 weeks: $\mathrm{n}=9$ for chow and HF-HSD groups, $\mathrm{n}=8$ for dietary intervention group.

more, HF-HSD did not affect mRNA levels of peroxisome proliferative activated receptor gamma coactivator 1 alpha (Ppargc1 $1 \alpha$ ), a gene involved in the regulation of mitochondrial biogenesis and BAT thermogenesis [22]. No difference in adrenergic receptor beta 3 (Adrb3), a gene involved in the regulation of lipolysis and thermogenesis after beta-adrenergic stimulation [23], was observed. After 20 weeks HF-HSD diet, BAT to body weight ratio was significantly increased compared to the NCD group ( $\vee$ Fig. $1 \mathbf{S}$ ).

\section{Gene expression related to lipid metabolism and energy expenditure in BAT 8 weeks after the initiation of the dietary intervention}

Of all the analyzed genes in mice that underwent a dietary intervention (normal liver histology), we only observed a significant increase in the BAT energy expenditure related gene Dio2 compared with mice that received a HF-HSD during 20 weeks (NASH) ( $\triangleright$ Fig. 2). All other genes concerning fatty acid metabolism ( $\vee$ Fig. 2a) and energy expenditure ( $\bullet$ Fig. $\mathbf{2 b}$ ) displayed no significant difference in mRNA expression levels. Dietary intervention completely restored the BAT to body weight ratio ( $\vee$ Fig. 1S).
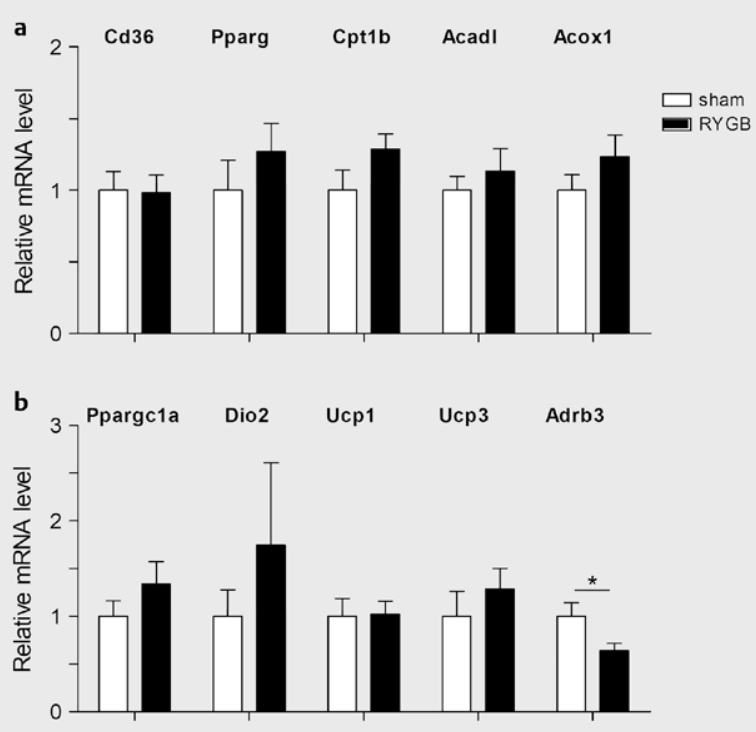

Fig. 3 Effect of sham or RYGB operation on genes related to BAT activity. a mRNA levels of genes involved in fat metabolism: Cluster of Differentiation $36(\mathrm{Cd} 36)$, peroxisome proliferator-activated receptor gamma (Ppary), Carnitine palmitoyltransferase 1b (Cpt1b) and the lipid beta-oxidation genes acyl-Coenzyme A dehydrogenase, long-chain (Acadl) and acyl-Coenzyme A oxidase 1 (Acox1). b mRNA levels of genes involved in energy expenditure: peroxisome proliferative activated receptor, gamma, coactivator 1 alpha (Ppargc1 $1 \alpha$ ), lodothyronine Deiodinase 2 (Dio2), Uncoupling Protein 1 (Ucp1), Uncoupling Protein 3 (Ucp3), adrenergic receptor beta 3 (Adrb3). ${ }^{*}: p<0.05$ and ${ }^{* *}: p<0.01$. Data are presented as mean. Error bars are standard error of mean (SEM).

\section{Gene expression related to lipid metabolism and energy expenditure in BAT 8 weeks after RYGB}

Mice that were fed a HF-HSD for 12 weeks (NASH) underwent a RYGB or SHAM procedure. Following surgery all mice received a HFHSD ad libitum for 8 weeks. No statistical difference in BAT gene expression regarding lipid metabolism was observed between the RYGB group and the SHAM group ( $\bullet$ Fig. 3a). Adrb3 gene expression was significantly lower in the RYGB operated mice while no difference in the expression of other energy expenditure genes was observed ( $\vee$ Fig. 3b).

\section{Gene expression levels of pro-inflammatory cytokines are increased in BAT after 20 weeks HF-HSD and are normalized by dietary intervention and RYGB}

Four-Weeks HF-HSD (no NASH) did not induce an increased gene expression of the pro-inflammatory cytokines II1 b, Tnf- $\alpha$ and MCP-1 in BAT ( Fig. 4a). After 20 weeks HF-HSD, the expression of these pro-inflammatory genes was significantly higher compared to mice that received a NCD for 20 weeks and mice that underwent the dietary intervention. In line with these results, plasma Tnf- $\alpha$ levels ( $\vee$ Table 3S) were under the lower limit of quantification in the vast majority of mice on NCD and mice that underwent a die- 

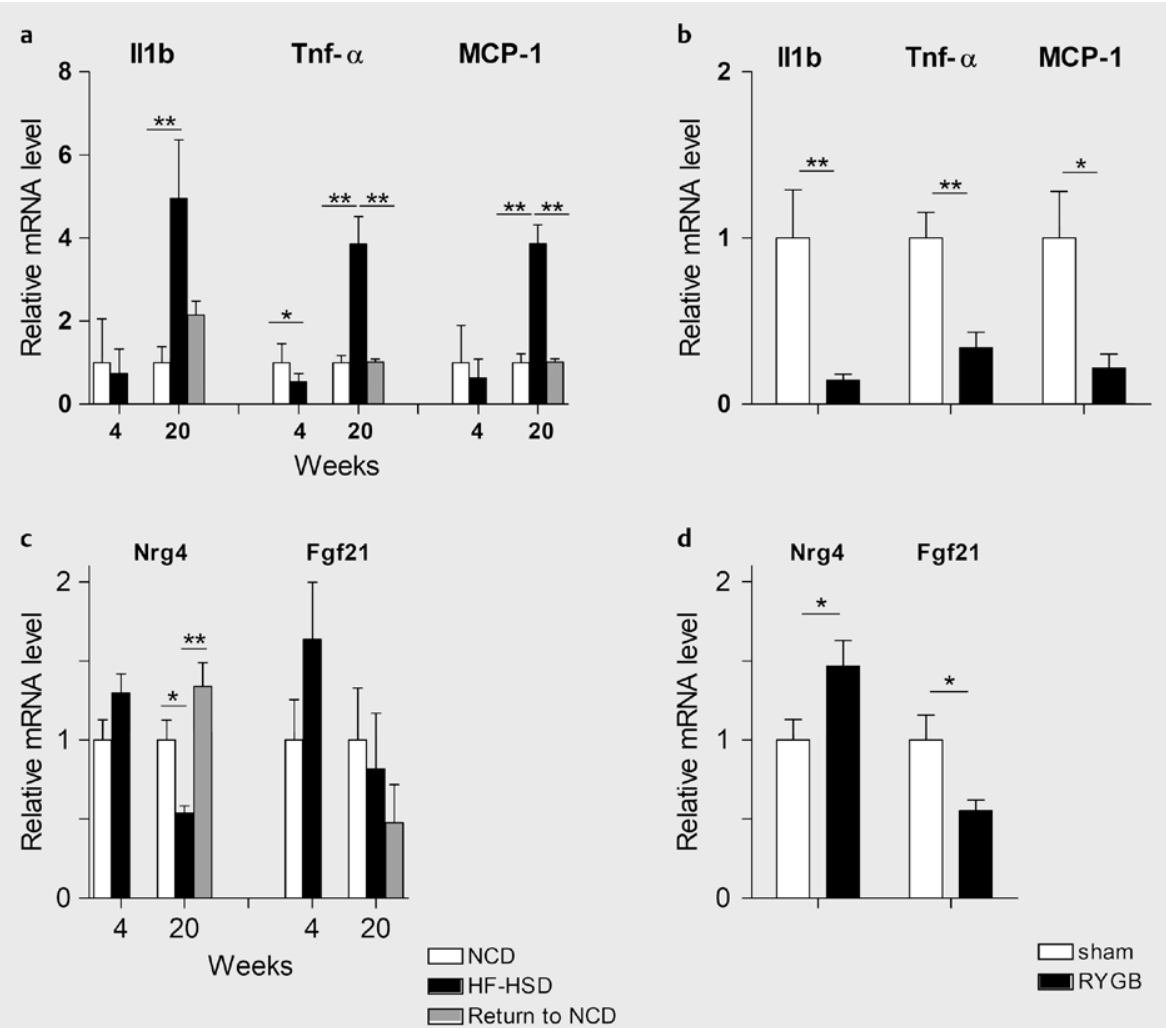

\begin{abstract}
- Fig. 4 Effect HF-HSD, dietary intervention and RYGB on gene expression levels of pro-inflammatory cytokines and batokines in BAT. a mRNA levels of pro-inflammatory cytokines in BAT of mice receiving a HF-HSD, NCD or dietary intervention. $\mathbf{b}$ mRNA levels of pro-inflammatory cytokines in BAT of mice receiving a RYGB or SHAM operation after 12 weeks HF-HSD. c mRNA levels of neuregulin 4 (Nrg4) and fibroblast growth factor 21 (Fgf21) in BAT of mice receiving a HF-HSD, NCD or dietary intervention. $\mathbf{d}$ mRNA levels of Nrg4 and Fgf21 in BAT of mice receiving a RYGB or SHAM operation after 12 weeks HF-HSD. ${ }^{*} \mathrm{p}<0.05$ and ${ }^{*} * \mathrm{p}<0.01$. Data are presented as mean. Error bars are standard error of mean (SEM). Interleukin 1 beta (II1ß); tumor necrosis factor alpha (Tnf- $\alpha$ ); monocyte chemoattractant protein 1 (MCP-1).
\end{abstract}

tary intervention, in contrast to the mice on HF-HSD after 4 weeks $(7.4 \pm 1.1 \mathrm{pg} / \mathrm{ml})$ and 20 weeks $(13.2 \pm 2.4 \mathrm{pg} / \mathrm{ml})$.

Gene expression levels II $1 \mathrm{~b}$, Tnf- $\alpha$ and MCP-1 were all significantly lower in BAT tissue of RYGB mice compared to SHAM mice ( $>$ Fig. 4b). Plasma Tnf- $\alpha$ levels were not significantly different between RYGB $(12.9 \pm 4.3 \mathrm{pg} / \mathrm{ml})$ and sham operated mice $(20.1 \pm$ $8.4 \mathrm{pg} / \mathrm{ml})(\mathrm{p}=0.39)$.

\section{$\mathrm{Nrg} 4$ gene expression in BAT is decreased after 20 weeks HF-HSD and is restored by dietary intervention and RYGB procedure}

$\mathrm{Nrg} 4$ and Fgf21 gene expression in BAT were analyzed in all dietary groups and time-points ( $\mathbf{F i g}$. 4c, d). Nrg4 expression did not change after 4-weeks HF-HSD, but was significantly lower after 20 weeks HF-HSD compared to NCD $(p<0.05)$. Both dietary intervention and RYGB procedure led to increased $\mathrm{Nrg} 4$ gene expression compared to 20 weeks HF-HSD or SHAM mice respectively $(p<0.01$ and $p<0.05$, respectively). Fgf2 1 gene expression was significantly decreased after RYGB compared to SHAM mice $(p<0.05)$.

\section{Discussion}

The absence of NASH after 4 weeks HF-HSD in our model, together with the occurrence of NASH after 20 weeks HF-HSD and the improvement of NASH after dietary intervention and RYGB $[17,18]$, allowed us to analyze BAT changes in the context of NASH development and after effective therapeutic interventions. Since molecular studies on BAT are hardly feasible in humans, we decided to use a representative mouse model. This mouse model closely mimics human NASH with respect to 1 ) the method of induction (a Western diet rich in sucrose and saturated fatty acids), 2) the systemic metabolic profile and 3) the histological spectrum of NASH $[17,18]$. In this exploratory study, we showed that the initial increase in expression of genes involved in lipid metabolism and energy expenditure in BAT is lost in the occurrence of NASH in mice receiving a HF-HSD. This finding might suggest that the loss of an initially increased BAT activity to compensate for an energy overload might contribute to the development of NASH. In addition, the gene expression profile of BAT was modulated by both dietary intervention and RYGB.

Our observation of an initial upregulation of BAT fatty acid oxidation during a high fat diet is in line with previous findings in other tissues including the liver $[17,24]$, heart and skeletal muscle 
[25-27], and is probably secondary to an increased supply of fatty acids. Similar to our findings, Poekes et al. observed in their study an increased BAT gene expression of Ucp 1 in wild type mice following a 4 weeks HFD (60\% of calories from fat) without any histological sign of hepatic steatosis [26]. Only one other study also evaluated BAT changes upon a high fat diet in a timeline manner [25]. Similar to our results, Ohtomo et al. observed an initial increase of Ucp 1 and carnitine palmitoyltransferase 2 mRNA levels after 4 weeks HFD, which was lost after 20 weeks HFD. However, in contrast to our study, histological analysis of the liver was not performed, thus the relationship with NASH (development) could not be assessed. In addition, we used a diet rich in not only saturated fatty acids but also in fructose, which has been identified as an independent risk factor for the development of NASH [28].

Our data are consistent with the concept of BAT whitening in the context of obesity after 20 weeks HF-HSD. It comprises a conversion of brown adipocytes to a white adipocyte-like phenotype. Although we could not perform histological analysis, BAT whitening is consistent with the increased BAT weight, increased expression of pro-inflammatory cytokines Tnf- $\alpha$, MCP-1 and II $1 \mathrm{~b}$ and unchanged expression of BAT energy expenditure genes after 20 weeks HF-HSD in our cohort $[29,30]$. The mechanism behind the loss in the upregulation of genes related to fatty acid oxidation and energy expenditure after a long-term HF-HSD feeding is unknown. This may be related to fatty acid overload itself in combination with factors in the plasma targeting BAT. Prolonged fatty acid overload, could impair mitochondrial function by accumulation of lipotoxic fatty acid intermediates in the mitochondria that cause inflammation, apoptosis and necrosis [31]. Chronic low-grade inflammation associated with the metabolic syndrome may harm BAT function. In line with this, we observed an increased gene expression in BAT of all investigated pro-inflammatory cytokines after 20 weeks HFHSD. In animal studies and in vitro experiments, both systemic inflammation and local BAT inflammation have been observed to reduce the induction of energy expenditure genes in BAT [32].

Dietary intervention resulted in a complete resolution of NASH, obesity and glucose intolerance without a change in BAT lipid metabolism and energy expenditure related genes.[18] Similarly, in our earlier report we did not observe significant changes in genes related to fatty acid oxidation and energy expenditure in the liver after dietary intervention in these mice. We hypothesize that the analysis of these BAT genes on an earlier time point than 8 weeks after the dietary intervention might reveal a significant difference in gene expression levels compatible with a clean-up of accumulating fatty acids by fatty acid oxidation and mitochondrial activity [18]. In contrast, BAT gene expression of pro-inflammatory cytokines normalized after dietary intervention, which is in line with the absence of NASH and thus liver inflammation and in line with our previously reported gene expression findings in the liver [18].

RYGB induced weight loss, improved insulin resistance and inhibited progression of NASH but did not induce a complete resolution of NASH [17]. In this experiment, RYGB operated mice had similar levels of lipid metabolism and energy expenditure mRNA levels in BAT compared to SHAM operated mice. In human studies, an increased resting metabolic rate (body weight adjusted) after RYGB is observed [33]. However, the exact mechanism and the contribution of BAT remains uncertain. Both browning of white adi- pose tissue and activation of brown adipose tissue has been described after a bariatric intervention [34]. In animal studies, this topic showed some contradictory results. Similar to our results, Hankir et al. observed that BAT activity and Ucp1 expression in RYGB operated obese Wistar rats did not significantly differ from ad libitum fed sham-operated controls 50 days after surgery [35]. In diet induced obese mice, Chen et al. observed a significant increase of BAT Ucp 1 mRNA expression 4 weeks post RYGB procedure, which declined 8 weeks post RYGB [36]. Because we only have gene expression data 8 weeks post RYGB, we might have missed the initial upregulation of Ucp 1 mRNA observed by Chen et al. The observed significant decrease in BAT Adrb3 gene expression following RYGB is in line with the findings of Abegg et al. who found a lower capacity of BAT thermogenesis following admission of a Adrb3 agonist in RYGB rats [37]. Adrb3 stimulation has been observed to cause a significant induction of Fgf21 mRNA levels in BAT [38]. The significant decrease in Adrb3 gene expression following RYGB might be related to the lower Fgf21 gene expression in our experiment.

BAT may counteract the development and progression of NAFLD by energy expenditure but also by the secretion of batokines that might impact the liver. Gene expression of Nrg4 was significantly decreased after 20 weeks HF-HSD. This is an interesting finding given the recent observation of a potential beneficial role of Nrg4 on hepatic lipogenesis and NASH $[39,40]$. Chen et al. observed a significantly decreased Nrg4 expression in cultured adipocytes with Tnf- $\alpha[41]$, suggesting a rate-limiting role of inflammatory stimuli $[42,43]$. Accordingly, we observed an increased II $1 \mathrm{~b}, \mathrm{Tnf}-\alpha$ and MCP-1 gene expression in BAT and increased plasma levels of Tnf- $\alpha$ after 20 weeks HF-HSD compared to 20 weeks NCD. In addition, for the first time we show an upregulation of $\mathrm{Nrg} 4$ gene expression in BAT after dietary intervention and RYGB. The observed increased Nrg4 expression in BAT after RYGB and dietary intervention co-occurred with a decreased gene expression of inflammatory cytokines II $1 \mathrm{~b}$, Tnf- $\alpha$ and MCP-1 in BAT. However, further studies are needed to clarify the upstream mediators, downstream effects and overall relevance of $\mathrm{Nrg} 4$ in human $\mathrm{NASH}$.

Despite the unique time-line and interventional set-up, this exploratory study has limitations. Protein, histological or functional analyses of BAT could have given valuable additional insights. However, our study can serve as a basis for further in-depth molecular studies on BAT in the context of NASH pathophysiology and treatment. All mice were sacrificed after 6 hours of fasting. It would be interesting to perform a similar gene expression analysis in mice in a non-fasting or fed state. It might be that the observed differences in lipid metabolism and energy expenditure would be even more pronounced.

In conclusion, our findings suggest that BAT mitochondrial function is enhanced after a short-term HF-HSD, probably as a compensatory response to process the fatty acid overload. Longer-term HF-HSD might induce the loss of this adaptive BAT function, which might contribute to the occurrence of NASH in these mice. Future studies with detailed profiling of BAT function, focused on both metabolic fluxes and endocrine factors, are needed to further decipher the dynamic role of BAT in NASH. Ultimately, interventional studies are needed to establish BAT as a direct therapeutic target in the treatment of NASH. 


\section{Funding Information}

This research was funded by a seeding grant of the School of Nutrition and Translational Research in Metabolism (NUTRIM), Maastricht University, The Netherlands.

\section{Conflict of Interest}

The authors declare that they have no conflict of interest.

\section{References}

[1] Younossi ZM, Koenig AB, Abdelatif D et al. Global epidemiology of nonalcoholic fatty liver disease-Meta-analytic assessment of prevalence, incidence, and outcomes. Hepatology 2016; 64: 73-84

[2] Lonardo A, Nascimbeni F, Mantovani A et al. Hypertension, diabetes, atherosclerosis and NASH: Cause or consequence? Journal of Hepatology 2018; 68: 335-352

[3] Calzadilla Bertot L, Adams LA. The natural course of non-alcoholic fatty liver disease. Int J Mol Sci 2016; 17: 774

[4] Wong R], Aguilar M, Cheung R et al. Nonalcoholic steatohepatitis is the second leading etiology of liver disease among adults awaiting liver transplantation in the United States. Gastroenterology 2015; 148: 547-555

[5] Sumida Y, Yoneda M. Current and future pharmacological therapies for NAFLD/NASH. J Gastroenterol 2018; 53: 362-376

[6] Byrne CD, Targher G. NAFLD: a multisystem disease. J Hepatol 2015; 62: $547-564$

[7] Scheja L, Heeren J. Metabolic interplay between white, beige, brown adipocytes and the liver. J Hepatol 2016; 64: 1176-1186

[8] Busiello RA, Savarese S, Lombardi A. Mitochondrial uncoupling proteins and energy metabolism. Front Physiol 2015; 6: 36

[9] Tutunchi H, Ostadrahimi A, Hosseinzadeh-Attar M-] et al. A systematic review of the association of neuregulin 4 , a brown fat-enriched secreted factor, with obesity and related metabolic disturbances. Obes Rev 2020; 21: e12952

[10] Liu J, Xu Y, Hu Y et al. The role of fibroblast growth factor 21 in the pathogenesis of non-alcoholic fatty liver disease and implications for therapy. Metabolism 2015; 64: 380-390

[11] Poekes L, Gillard J, Farrell GC et al. Activation of brown adipose tissue enhances the efficacy of caloric restriction for treatment of nonalcoholic steatohepatitis. Lab Invest 2019; 99: 4-16

[12] van Marken Lichtenbelt WD, Vanhommerig JW, Smulders NM et al. Cold-activated brown adipose tissue in healthy men. $\mathrm{N}$ Eng J Med 2009; 360: 1500-1508

[13] Cypess AM, Lehman S, Williams $G$ et al. Identification and Importance of Brown Adipose Tissue in Adult Humans. N Eng J Med 2009; 360: 1509-1517

[14] Yilmaz Y, Ones T, Purnak T et al. Association between the presence of brown adipose tissue and non-alcoholic fatty liver disease in adult humans. Aliment Pharmacol Ther 2011; 34: 318-323

[15] Baskin AS, Linderman JD, Brychta RJ et al. Regulation of human adipose tissue activation, gallbladder size, and bile acid metabolism by a beta3-adrenergic receptor agonist. Diabetes 2018; 67: 2113-2125

[16] Verbeek J, Jacobs A, Spincemaille P et al. Development of a representative mouse model with nonalcoholic steatohepatitis. Curr Protoc Mouse. Biol 2016; 6: 201-210
[17] Verbeek J, Lannoo M, Pirinen E et al. Roux-en-y gastric bypass attenuates hepatic mitochondrial dysfunction in mice with non-alcoholic steatohepatitis. Gut 2015; 64: 673-683

[18] Verbeek J, Spincemaille P, Vanhorebeek I et al. Dietary intervention, but not losartan, completely reverses non-alcoholic steatohepatitis in obese and insulin resistant mice. Lipids Health Dis 2017; 16: 46

[19] Bartelt A, Bruns OT, Reimer $R$ et al. Brown adipose tissue activity controls triglyceride clearance. Nat Med 2011; 17: 200

[20] Townsend KL, Tseng Y-H. Brown Fat Fuel Utilization and Thermogenesis. Trends Endocrinol Metab 2014; 25: 168-177

[21] Arrojo EDR, Fonseca TL, Werneck-de-Castro JP et al. Role of the type 2 iodothyronine deiodinase (D2) in the control of thyroid hormone signaling. Biochim Biophys Acta 2013; 1830: 3956-3964

[22] Gill JA, La Merrill MA. An emerging role for epigenetic regulation of Pgc-1 $\alpha$-expression in environmentally stimulated brown adipose thermogenesis. Environ Epigenet 2017; 3: dvx009

[23] Hu J, Zhou H, Smyth A et al. Polymorphism of the bovine ADRB3 gene. Mol Biol Rep 2010; 37: 3389-3392

[24] Chan MY, Zhao Y, Heng CK. Sequential responses to high-fat and high-calorie feeding in an obese mouse model. Obesity (Silver Spring) 2008; 16: 972-978

[25] Ohtomo T, Ino K, Miyashita R et al. Chronic high-fat feeding impairs adaptive induction of mitochondrial fatty acid combustion-associated proteins in brown adipose tissue of mice. Biochem Biophys Rep 2017; 10: $32-38$

[26] Poekes L, Legry V, Schakman O et al. Defective adaptive thermogenesis contributes to metabolic syndrome and liver steatosis in obese mice. Clin Sci (Lond) 2017; 131: 285-296

[27] Fujita M, Momose A, Ohtomo T et al. Upregulation of fatty acyl-CoA thioesterases in the heart and skeletal muscle of rats fed a high-fat diet. Biol Pharm Bull 2011; 34: 87-91

[28] Mosca A, Nobili V, De Vito R et al. Serum uric acid concentrations and fructose consumption are independently associated with $\mathrm{NASH}$ in children and adolescents. J Hepatol 2017; 66: 1031-1036

[29] Roberts-Toler C, O'Neill BT, Cypess AM. Diet-induced obesity causes insulin resistance in mouse brown adipose tissue. Obesity (Silver Spring, Md) 2015; 23: 1765-1770

[30] Dinh CHL, Szabo A, Yu Y et al. Bardoxolone methyl prevents fat deposition and inflammation in brown adipose tissue and enhances sympathetic activity in mice fed a high-fat diet. Nutrients 2015; 7: $4705-4723$

[31] Koves TR, Ussher JR, Noland RC et al. Mitochondrial overload and incomplete fatty acid oxidation contribute to skeletal muscle insulin resistance. Cell Metab 2008; 7: 45-56

[32] Nøhr MK, Bobba N, Richelsen B et al. Inflammation Downregulates UCP1 Expression in Brown Adipocytes Potentially via SIRT1 and DBC1 Interaction. Int J Mol Sci 2017; 18: 1006

[33] Faria SL, Faria OP, Buffington C et al. Energy expenditure before and after Roux-en-Y gastric bypass. Obes Surg 2012; 22: 1450-1455

[34] GHEJ Vijgen, Bouvy ND, G]J Teule et al. Increase in Brown Adipose Tissue Activity after Weight Loss in Morbidly Obese Subjects. J Clin Endocrinol Metab 2012; 97: E1229-E1233

[35] Hankir M, Bueter M, Gsell W et al. Increased energy expenditure in gastric bypass rats is not caused by activated brown adipose tissue. Obes Facts 2012; 5: 349-358

[36] Chen Y, Yang J, Nie X et al. Effects of Bariatric Surgery on Change of Brown Adipocyte Tissue and Energy Metabolism in Obese Mice. Obes Surg 2018; 28: 820-830

[37] Abegg K, Corteville C, Bueter M et al. Alterations in energy expenditure in Roux-en-Y gastric bypass rats persist at thermoneutrality. Int J Obes 2016; 40: 1215 
[38] Chartoumpekis DV, Habeos IG, Ziros PG et al. Brown adipose tissue responds to cold and adrenergic stimulation by induction of FGF21. Mol Med 2011; 17: 736-740

[39] Wang GX, Zhao XY, Meng ZX et al. The brown fat-enriched secreted factor Nrg4 preserves metabolic homeostasis through attenuation of hepatic lipogenesis. Nat Med 2014; 20: 1436-1443

[40] Guo L, Zhang P, Chen Z et al. Hepatic neuregulin 4 signaling defines an endocrine checkpoint for steatosis-to-NASH progression. J Clin Invest 2017; 127: 4449-4461
[41] Chen Z, Wang G-X, Ma SL et al. Nrg4 promotes fuel oxidation and a healthy adipokine profile to ameliorate diet-induced metabolic disorders. Mol Metab 2017; 6: 863-872

[42] Rao SR. Inflammatory markers and bariatric surgery: A meta-analysis. Inflamm Res 2012; 61: 789-807

[43] Christiansen T, Paulsen SK, Bruun JM et al. Exercise training versus diet-induced weight-loss on metabolic risk factors and inflammatory markers in obese subjects: a 12-week randomized intervention study. Am J Physiol Endocrinol Metab 2010; 298: E824-E831 
Supplementary Material

\section{The Role of Brown Adipose Tissue in the Development and Treatment of Nonalcoholic Steatohepatitis: An Exploratory Gene Expression Study in Mice}

\section{Authors}

Toon J.I. De Munck ${ }^{1,2}$, Pan Xu ${ }^{1,2}$, Brechtje L.J. Vanderfeesten ${ }^{1}$, Montserrat Elizalde ${ }^{2}$, Ad A.M. Masclee ${ }^{1,2}$, Frederik Nevens ${ }^{3}$, David Cassiman ${ }^{3}$, Frank G. Schaap ${ }^{2,4,5}$, Daisy M.A.E. Jonkers ${ }^{1,2}$, jef Verbeek ${ }^{3}$

\section{Affiliations}

${ }^{1}$ Division of Gastroenterology and Hepatology, Department of Internal Medicine, Maastricht University Medical Centre, Maastricht, The Netherlands

${ }^{2}$ School of Nutrition and Translational Research in Metabolism (NUTRIM), Maastricht University, The Netherlands

${ }^{3}$ Department of Gastroenterology and Hepatology, University Hospitals KU Leuven, Leuven, Belgium

${ }^{4}$ Department of Surgery, Maastricht University Medical Centre, Maastricht, The Netherlands

${ }^{5}$ Department of General, Visceral and Transplantation Surgery, RWTH University Hospital Aachen, Aachen, Germany.

\section{Correspondence}

Jef Verbeek, MD, PhD

Department of Gastroenterology and Hepatology, University Hospitals KU Leuven Herestraat 49, Leuven, Belgium

Tel.: + 3216344225

jef.verbeek@uzleuven.be 
Table 1S: NAFLD Activity Scores (NAS) of mice in each study group as previously published [1, 2]

\begin{tabular}{|c|c|c|c|c|c|c|c|}
\hline & \multicolumn{2}{|c|}{ Experiment $A$} & \multicolumn{3}{|c|}{ Experiment B } & \multicolumn{2}{|c|}{ Experiment $\mathrm{C}$} \\
\hline & $\begin{array}{l}\text { NCD } \\
4 \mathrm{wk}\end{array}$ & $\begin{array}{c}\text { HF-HSD } \\
4 \text { wk }\end{array}$ & $\begin{array}{l}\text { NCD } \\
20 w k\end{array}$ & $\begin{array}{l}\text { HF-HSD } \\
20 \text { wk }\end{array}$ & $\begin{array}{l}\text { Return } \\
\text { to NCD }\end{array}$ & Sham & RYGB \\
\hline Steatosis & $0.2 \pm 0.2$ & $0.2 \pm 0.2$ & $0.0 \pm 0.0$ & $2.9 \pm 0.1$ & $0.0 \pm 0.0$ & $2.8 \pm 0.2$ & $1.2 \pm 0.4$ \\
\hline $\begin{array}{l}\text { Hepatocyte } \\
\text { Ballooning }\end{array}$ & $0.1 \pm 0.1$ & $0.0 \pm 0.0$ & $0.0 \pm 0.0$ & $1.2 \pm 0.1$ & $0.0 \pm 0.0$ & $1.8 \pm 0.2$ & $1.5 \pm 0.2$ \\
\hline Inflammation & $0.0 \pm 0.0$ & $0.0 \pm 0.0$ & $0.0 \pm 0.0$ & $1.2 \pm 0.1$ & $0.0 \pm 0.0$ & $2.0 \pm 0.3$ & $1.0 \pm 0.0$ \\
\hline Fibrosis & $0.0 \pm 0.0$ & $0.0 \pm 0.0$ & $0.0 \pm 0.0$ & $0.9 \pm 0.2$ & $0.0 \pm 0.0$ & $1.8 \pm 0.2$ & $0.0 \pm 0.0$ \\
\hline NAS & $0.4 \pm 0.4$ & $0.2 \pm 0.2$ & $0.0 \pm 0.0$ & $5.3 \pm 0.2$ & $0.0 \pm 0.0$ & $6.6 \pm 0.7$ & $3.7 \pm 0.3$ \\
\hline
\end{tabular}

Data are presented as mean and SEM; Steatosis was scored as 0 (<5\%), 1 (5-33\%), 2 (>33-66\%) and 3 (>66\%). Hepatocyte ballooning was classified as 0 (none), 1 (few) or 2 (many cells/prominent ballooning). Foci of lobular inflammation were scored as 0 (no foci), 1 (<2 foci per $200 \times$ field), 2 (2-4 foci per 200× field) and 3 ( $>4$ foci per $200 \times$ field). Fibrosis was scored as stage F0 (no fibrosis), stage F1a (mild, zone 3 , perisinusoidal fibrosis), stage F1b (moderate, zone 3, perisinusoidal fibrosis), stage F1c (portal/periportal fibrosis), stage F2 (perisinusoidal and portal/periportal fibrosis), stage F3 (bridging fibrosis) and stage F4 (cirrhosis). [1] Verbeek J, Lannoo M, Pirinen E et al. Roux-en-y gastric bypass attenuates hepatic mitochondrial dysfunction in mice with non-alcoholic steatohepatitis. Gut 2015; 64: 673-683; [2] Verbeek J, Spincemaille P, Vanhorebeek I et al. Dietary intervention, but not losartan, completely reverses non-alcoholic steatohepatitis in obese and insulin resistant mice. Lipids Health Dis 2017; 16: 46 
Table 2S Primer sets for real time PCR analysis of gene expression.

\begin{tabular}{|l|l|l|}
\hline Name & Forward sequence & Reverse sequence \\
\hline Cyclophilin A & TTCCTCCTTTCACAGAATTATTCCA & CCGCCAGTGCCATTATGG \\
\hline Cd36 & GATGTGGAACCCATAACTGGATTCAC & GGTCCCAGTCTCATTTAGCCACAGTA \\
\hline Ucp1 & CTTTGCCTCACTCAGGATTGG & ACTGCCACACCTCCAGTCATT \\
\hline Ucp3 & CTGCACCGCCAGATGAGTTT & TCCAAAGGCAGAGACAAAGTG A \\
\hline Cpt1b & CCCATGTGCTCCTACCAGAT & CCTTGAAGAAGCGACCTTTG \\
\hline Dio2 & AGACTTGCTGATCACCCTGC & ACCACACTGGAATTGGGAGC \\
\hline Acox1 & GCCCAACTGTGACTTCCATC & GGCATGTAACCCGTAGCACT \\
\hline Ppargc1a & TGAGGACCGCTAGCAAGTTT & TGTAGCGACCAATCGGAAAT \\
\hline Adrb3 & ACAGCAGACAGGGACAGAGG & TCCTGTCTTGACACTCCCTCA \\
\hline Pparg & AGGCCGAGAAGGAGAAGCTGTTG & TGGCCACCTCTTTGCTCTGCTC \\
\hline Tnf- $\alpha$ & TTTGAGATCCATGCCGTTG & CTGTAGCCCACGTCGTAGC \\
\hline Il1b & GCCCATCCTCTGTGACTCAT & AGGCCACAGGTATTTTGTCG \\
\hline MCP-1 & GCTGGAGAGCTACAAGAGGATCA & CTCTCTCTTGAGCTTGGTGACAAA \\
\hline Acadl & GTAGCTTATGAATGTGTGCAACTC & GTCTTGCGATCAGCTCTTTCATTA \\
\hline Nrg4 & CCCAGCCCATTCTGTAGGTG & ACCACGAAAGCTGCCGACAG \\
\hline Fgf21 & GCTGCTGGAGGACGGTTACA & CACAGGTCCCCAGGATGTTG \\
\hline
\end{tabular}

Table 35 Plasma Tnf- $\alpha$ in all dietary groups

\begin{tabular}{|c|c|c|}
\hline & & $\begin{array}{l}\text { Plasma Tnf- } \alpha(\mathrm{pg} / \mathrm{ml}) \\
(L L O Q=5 \mathrm{pg} / \mathrm{ml})\end{array}$ \\
\hline \multirow[t]{2}{*}{ Experiment $\mathrm{A}$} & 4 week HF-HSD $(n=4)$ & $7.4 \pm 1.1$ \\
\hline & 4 week NCD $(n=4)$ & 4/4 cases below LLOQ \\
\hline \multirow[t]{3}{*}{ Experiment B } & 20 week HF-HSD $(n=9)$ & $13.2 \pm 2.4$ \\
\hline & Return to NCD (=5) & 3/5 cases below LLOQ \\
\hline & 20 week NCD $(n=7)$ & 6/7 cases below LLOQ \\
\hline \multirow[t]{2}{*}{ Experiment $\mathrm{C}$} & RYGB (n=6) & $12.9 \pm 4.3$ \\
\hline & Sham $(n=6)$ & $20.1 \pm 8.4$ (NS) \\
\hline
\end{tabular}

Values are expressed as mean \pm SEM. LLOQ: Lower limit of quantification 


\section{BAT weight / body weight}

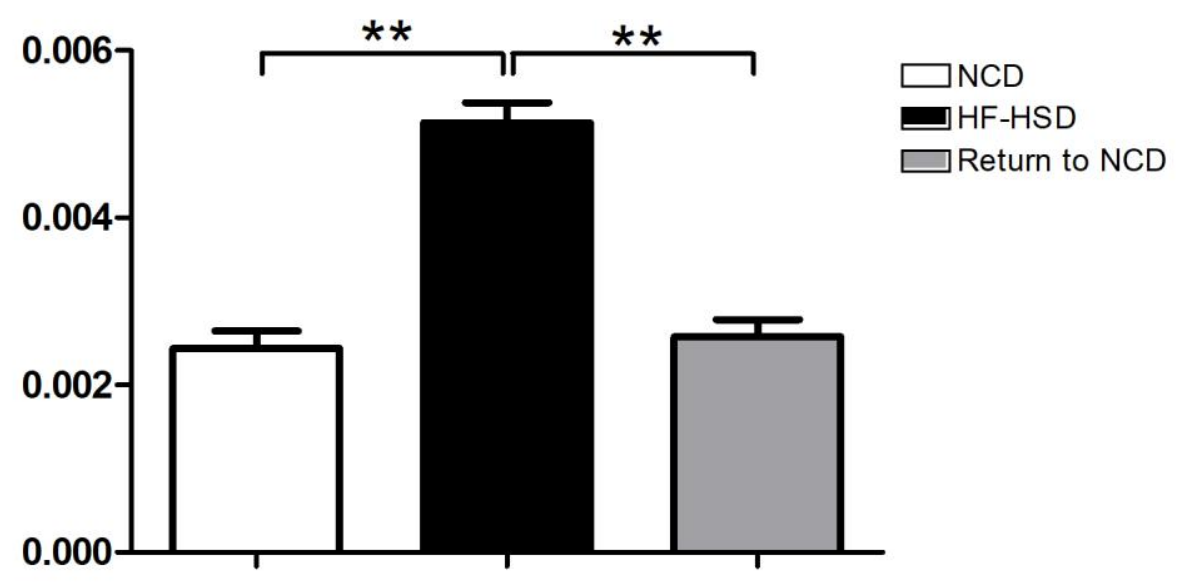

Figure 1S. The effect of 20 weeks HF-HSD and dietary intervention on BAT weight / body weight ratio. ${ }^{*}: \mathrm{P}<0.05$ and $^{* *}: \mathrm{P}<0.01$. Data are presented as mean. Error bars are standard error of mean (SEM). 